\title{
AUTOMATING SYMMETRY-BREAKING CALCULATIONS
}

\section{P. C. MATTHEWS}

\begin{abstract}
The process of classifying possible symmetry-breaking bifurcations requires a computation involving the subgroups and irreducible representations of the original symmetry group. It is shown how this calculation can be automated using a group theory package such as GAP. This enables a number of new results to be obtained for larger symmetry groups, where manual computation is impractical. Examples of symmetric and alternating groups are given, and the method is also applied to the spatial symmetry-breaking of periodic patterns observed in experiments.
\end{abstract}

\section{Introduction}

This paper is concerned with symmetry-breaking bifurcations, in which a state with a high degree of symmetry becomes unstable to a state with less symmetry. As an introductory example, consider an object with symmetry (for example, spherical, cubic or square symmetry) subjected to steadily increasing external pressure. As the pressure increases, the object may retain its symmetry, or, at a critical value of the pressure, it may spontaneously buckle into a different shape with less symmetry. More generally, symmetry-breaking bifurcations occur in systems of ordinary or partial differential equations with a certain symmetry; there is a solution of the equations with this full symmetry, but this state may become unstable as a parameter is varied. Because of the symmetry, the linear stability problem has multiple eigenvalues, with a multiplicity given by the dimension of one of the irreducible representations of the symmetry group. This means that the linear stability problem is degenerate, and this degeneracy can be resolved only by considering the nonlinear terms in the equations, which are themselves constrained by the symmetry. The general theory for such transitions is set out in the key text on the subject by Golubitsky, Stewart and Schaeffer [11], building on earlier work by Sattinger [22]; two more recent books incorporate newer theoretical results and provide many examples and applications of the theory $[4,10]$.

A remarkable feature of such problems is that the possible symmetry-breaking transitions can be determined purely from the symmetry of the problem, independently of the underlying physical mechanisms involved, by the use of group and representation theory. However, in order to determine which of the possible transitions lead(s) to a stable solution in a particular application, it is necessary to return to the original equations and compute the values of certain coefficients.

One application of considerable interest is the formation and stability of patterns. In experiments on Rayleigh-Bénard convection, where a layer of fluid is heated from below, and in the Faraday experiment, where a layer of fluid or granular material is subjected to a vertical oscillation, the original Euclidean symmetry can be broken, leading to a regular 
pattern of stripes, squares or hexagons $[21,3, \mathbf{1}]$. These regular periodic patterns may themselves become unstable, leading to secondary patterns that may retain periodicity on a lengthscale longer than that of the original pattern $[14,24,6,7]$.

The aim of this paper is to show how the basic group-theoretic approach to classifying possible symmetry-breaking bifurcations can be straightforwardly automated by using a computational group theory package such as GAP [8]. Section 2 summarises the general theory of the subject, and Section 3 describes the computational implementation. A number of results are presented in tabular form in Section 4, and these are extended to the case of oscillatory bifurcations in Section 5.

\section{Problem formulation}

This section introduces the language and notation of bifurcation with symmetry, and summarises the key results; full details can be found in [11] or [4]. Suppose that $G$ is a compact Lie group, acting absolutely irreducibly on $V=\mathbb{R}^{n}$. The representation is said to be absolutely irreducible if the only matrices that commute with all elements of $G$ are multiples of the identity, and this implies that the representation is irreducible (that is, there is no proper $G$-invariant subspace).

The system of $n$ equations

$$
f(x, \lambda)=0, \quad x \in \mathbb{R}^{n}, \lambda \in \mathbb{R}
$$

is said to be $G$-equivariant if

$$
f(g x, \lambda)=g f(x, \lambda) \quad \text { for all } g \in G,
$$

where the notation $g x$ is used as an abbreviation for the action of $g$ on $x$ through the matrix representation of $g$.

The symmetry of a point $v$ in $V$ is described by its isotropy subgroup, or stabiliser, defined by

$$
\{g \in G: g v=v\} .
$$

A subgroup $H \subset G$ is said to be an isotropy subgroup of $G$ if there exists a point $v \in V$ with isotropy $H$. In the physics literature, isotropy subgroups are often referred to as little groups. For any subgroup $H \subset G$, the fixed-point subspace of $H$ is

$$
\operatorname{Fix}(H)=\{v \in V: h v=v \text { for all } h \in H\} .
$$

The irreducibility condition means that either $\operatorname{Fix}(G)=V$ or $\operatorname{Fix}(G)=0$. However, if $\operatorname{Fix}(G)=V$, then any subspace of $V$ is $G$-invariant, which contradicts the irreducibility if $n>1$. Hence for $n>1$, we must have $\operatorname{Fix}(G)=0$.

We now suppose that the system of equations (1) has a solution that undergoes a stationary bifurcation at $x=0, \lambda=0$, so that

$$
f(0,0)=0 \quad \text { and } \quad\left|\frac{\partial f_{i}}{\partial x_{j}}\right|(0,0)=0 .
$$

The second condition, that the Jacobian matrix has zero determinant, means that the implicit function theorem does not hold, and so (1) does not define a unique branch of solutions $x(\lambda)$ in the vicinity of $\lambda=0$. The linearisation of $f$ must be a scalar multiple $c(\lambda)$ of the identity (from absolute irreducibility) with $c(0)=0$, and it is assumed that the transversality condition $c^{\prime}(0) \neq 0$ holds. 
The type of the bifurcation depends on the choice of representation of $G$. For the trivial representation, in which $g x=x$ for all $g$ and $x$, the equivariance condition (2) provides no constraint, and generically the bifurcation is of saddle-node type, in which the leading terms are, after a suitable rescaling, $\lambda+x^{2}=0$. There is no loss of symmetry at the bifurcation. For all other one-dimensional representations, $g x=-x$ for some $g$, and so (2) forces $f$ to be an odd function of $x$, and the bifurcation is of pitchfork type, $\lambda x+a x^{3}=0$ to leading order. The nonzero solutions have an isotropy subgroup generated by the elements of $G$ that act as +1 .

For irreducible representations (irreps) of dimension $n>1$, we must have $\operatorname{Fix}(G)=0$, as explained above. Applying (2) to $x=0$ gives $f(0, \lambda)=g f(0, \lambda)$ for all $g \in G$, so that $f(0, \lambda) \in \operatorname{Fix}(G)$, and hence $f(0, \lambda)=0$. Physically, this is the statement that a solution with the full symmetry group $G$ exists for all values of the parameter $\lambda$. The existence of non-zero solutions in this case can be established using the equivariant branching lemma $[5,25,13,11]$, which states that if, in addition to the conditions given above, there is an isotropy subgroup $H$ of $G$ with $\operatorname{Dim}(\operatorname{Fix}(H))=1$, then a unique branch of solutions to (1) with isotropy $H$ exists, emanating from $x=0, \lambda=0$. This condition is sufficient but not necessary, so applying the lemma in general finds some but not all of the solution branches of (1). The notation $D(H)$ will be used henceforth as an abbreviation for $\operatorname{Dim}(\operatorname{Fix}(H))$. If $D(H)>1$, there is no simple analogue of the equivariant branching lemma; however, if $D(H)=2$, then at least one solution exists in $\operatorname{Fix}(H)$, provided that the quadratic terms in the equations are non-zero and satisfy certain non-degeneracy conditions $[\mathbf{1 5}, \mathbf{1 6}, \mathbf{1 8}]$.

In the case where $n>1$, the type of bifurcation is constrained by the normalizer, $N(H)$, defined by

$$
N(H)=\left\{g \in G: g^{-1} H g=H\right\} .
$$

Equivalently, this is the largest subgroup of $G$ in which $H$ is normal. The normalizer maps Fix $(H)$ to itself, since if $y \in \operatorname{Fix}(H), g \in N(H)$ and $h \in H$, then $\left(g^{-1} h g\right) y=y$ (since $\left.g^{-1} h g \in H\right)$, so $h g y=g y$ and hence $g y \in \operatorname{Fix}(H)$. Clearly, $N(H)$ contains $H$, and if $N(H)=H$ then there is no symmetry within Fix $(H)$, since all elements of $H$ act as the identity in $\operatorname{Fix}(H)$. But if $N(H) \neq H$, then the symmetry group acting on $\operatorname{Fix}(H)$ is the quotient group $N(H) / H$. If $D(H)=1$, the only possibilities are either that $N(H)=H$, or that $N(H) / H$ is isomorphic to the cyclic group $Z_{2}$; in the latter case, the bifurcation is of pitchfork type. If $N(H)=H$, then the bifurcation is generally transcritical, but in some cases there may be additional constraints, for example due to 'hidden' symmetries $[10,7]$. A standard example is the group $D_{5}$, where $N(H)=H$ but the bifurcation is not transcritical.

In order to apply the equivariant branching lemma and hence obtain solutions of (1), one must find the isotropy subgroups $H$ for which $D(H)=1$. These are often referred to as axial subgroups, since they correspond to axes of reflection for two-dimensional irreps, and to axes of rotation for three-dimensional irreps (for the cube, for example, there are three such axes, with isotropies $Z_{4}, Z_{3}$ and $Z_{2}$ ). To compute $D(H)$, it is not necessary to have an explicit form of the matrix representation, since one can use the trace formula $[\mathbf{1 1}, \mathbf{4}]$

$$
D(H)=\operatorname{Dim}(\operatorname{Fix}(H))=\frac{1}{|H|} \sum_{h \in H} \chi(h),
$$

where $\chi(h)$ is the character of $h$ (the trace of the matrix). Thus the computation of $D(H)$ requires only the character table of $G$. 


\section{Computational algorithm}

The algorithm for computing the isotropy subgroups of a given finite group $G$ is outlined in Table 1. This algorithm was implemented using the computational group theory package GAP [8].

The first step is to define the group $G$, by specifying the generators of $G$. In most cases, the most convenient way to do this is to write $G$ as a subgroup of a permutation group, which can always be done (this is Cayley's theorem). For example, the group $D_{4}$ of symmetries of a square is expressed as a subgroup of $S_{4}$ by labelling the four corners; the two reflections that generate $D_{4}$ are then the permutations $(1,2)(3,4)$ and $(1,3)$. The next stage is to find the lattice of subgroups of $G$, up to conjugacy. Two subgroups $H$ and $K$ are said to be conjugate if there exists $g \in G$ such that $H=g^{-1} K g$; for example, the subgroups generated by $(1,3)$ and $(2,4)$ are conjugate, both corresponding to diagonal reflections of the square. If two subgroups are conjugate, then the dimensions of their fixed-point subspaces are equal. Note that two subgroups may be isomorphic but not conjugate; for example, $D_{4}$ has three nonconjugate subgroups that are isomorphic to $Z_{2}$. For each subgroup $H$, it is useful to check whether $H$ is normal, and to find the normaliser of $H$.

The next stage is to find the character table of $G$. Note that in a high-level language such as GAP, this is done in a single command (as is the construction of the subgroup lattice). We now choose one of the irreps of $G$, and calculate $D(H)$ for all non-conjugate subgroups of $G$ using the trace formula (6). In the GAP language, this is evaluated as a scalar product of the restriction of the character to $H$ with the trivial character of $H$. Having found the dimensions corresponding to each subgroup, the isotropy subgroups can be found, using

Table 1: Computational algorithm for finding isotropy subgroups.

Specify the group $G$ as a subgroup of a permutation group

Compute the lattice of non-conjugate subgroups of $G$

Compute the character table of $G$

For each irrep of $G$

For each subgroup $H$ of $G$

Compute $D(H)$ using trace formula

End for

For each subgroup $H$ of $G$

If $D(H)>0$ and $D(K)<D(H)$ for all $K$ immediately

above $H$ in the lattice, then $H$ is an isotropy subgroup

If $D(H)>0$ and $D(K)=0$ for all $K$ immediately

above $H$ in the lattice, then $H$ is maximal

If $H$ is an isotropy subgroup, find $N(H) / H$

End for

List isotropy subgroups $H$ and $D(H)$ for this irrep

End for 
the chain criterion (see, for example, [20]). This states that $H$ is an isotropy subgroup of $G$ if and only if $D(K)<D(H)$ for all $K \supsetneq H$. Since $K \supsetneq H \Rightarrow D(K) \leqslant D(H)$, it is only necessary to check whether $D(K)<D(H)$ for all $K$ immediately above $H$ in the subgroup lattice. Note that this chain criterion is valid only for finite groups; refinements have been proposed to provide a criterion for infinite groups, such as $\mathrm{SO}(3)$ and $O(3)$; see $[13,17]$. The group $G$ is itself always an isotropy subgroup, with $D(G)=0$ for all non-trivial irreps. Any subgroup $H \subsetneq G$ with $D(H)=0$ cannot be an isotropy subgroup, by the chain criterion, so it is only necessary to find isotropy subgroups with $D(H) \geqslant 1$. Having found an isotropy subgroup, it is useful to compute the quotient group $N(H) / H$, since this gives the symmetry acting on $\operatorname{Fix}(H)$, which influences the type of bifurcation.

Amongst isotropy subgroups, of particular interest are the maximal isotropy subgroups. An isotropy subgroup $H$ is said to be maximal if there is no isotropy subgroup $K$ satisfying $H \subsetneq K \subsetneq G$. Equivalently, $H$ is maximal if $D(H)>0$ and $D(K)=0$ for all $K$ immediately above $H$ in the subgroup lattice. Thus isotropy subgroups with $D(H)=1$ are always maximal, but those with $D(H)>1$ may or may not be maximal. Having found the isotropy subgroups for a given irrep, the computation is then repeated for the other irreps of $G$.

There are a number of short cuts that can be taken to reduce the calculations required. These may be useful when the calculation is being carried out by hand, but it turns out that there is little to be gained from implementing these computationally, since the saving in computational time is not significant. The most time-consuming part of the computation is the construction of the subgroup lattice; applying the trace formula and the chain criterion typically occupies less than one fifth of the computation time.

The first short cut is that it is really only necessary to consider faithful irreps (recall that an irrep is faithful if only the identity element of $g$ satisfies $g v=v$ for all $v \in V$ ). If the irrep is not faithful, the calculation can be reduced to the case of a smaller group, which is the quotient group $G / N$, where $N$ is the normal subgroup of $G$ containing the elements corresponding to the identity matrix. A standard example (see [11]) is the two-dimensional irrep of $S_{4}$, in which a normal subgroup isomorphic to $D_{2}$ acts as the identity and the quotient group is isomorphic to $S_{3}$. Faithful irreps can be identified from the character table from the fact that all (non-identity) conjugacy classes have a character less than that of the identity. For a faithful irrep of dimension $n$, it follows that the identity subgroup $I$ is an isotropy subgroup with $D(I)=n$, while for a non-faithful irrep, the above normal subgroup $N$ is an isotropy subgroup with $D(N)=n$.

The second short cut is that for faithful irreps, normal subgroups can never be isotropy subgroups. This simple result does not appear to be given explicitly in the standard literature, so it is formally stated and proved here.

LEMMA 1. Suppose that $G$ acts irreducibly and faithfully on $\mathbb{R}^{n}$, and that $H$ is a proper normal subgroup of $G$. Then $D(H)=0$, and so $H$ is not an isotropy subgroup of $G$.

Proof. If $H$ is a normal subgroup of $G$, then the normaliser of $H$ is $N(H)=G$, by definition. Then, as shown in Section 2, $G$ maps Fix $(H)$ to itself, so the subspace $\operatorname{Fix}(H)$ is $G$-invariant. Since the action of $G$ is irreducible, this subspace must be either $\mathbb{R}^{n}$ or 0 . Now if $\operatorname{Fix}(H)=\mathbb{R}^{n}$ and $H$ is not the identity subgroup, then there are non-identity elements that act as the identity, so the action cannot be faithful. Hence if the action is faithful, $\operatorname{Fix}(H)=\{0\}$, and so $D(H)=0$ and $H$ is not an isotropy subgroup. 
Thirdly, it is in fact not necessary to evaluate $D(H)$ for every group in the lattice. For example, once a subgroup with $D(H)=0$ has been identified, all the subgroups above $H$ in the lattice must have $D(H)=0$. Once again, there is no point in incorporating this into the computational algorithm.

A potentially useful extension of the calculation, beyond the scope of this paper, would be to construct the equations (1) that are consistent with the equivariance condition (2). This would permit automation of the explicit construction of solutions, which is of particular interest in cases where $D(H)$ is even, when solutions may or may not exist. This is a more difficult calculation, since it requires explicit construction of a matrix representation of $G$, which is non-unique, followed by the solution of a large number of equations, obtained from (2), relating the coefficients of nonlinear terms in (1).

\section{Results}

In this section, results are presented in tabular form, giving the isotropy subgroups for various finite groups of practical interest. Many of these results are new, although in some cases the results are available in the literature $[11,19,12,2]$. In each case below, reference is made, where appropriate, to earlier work.

In some cases, general results can be obtained analytically. For the group $D_{n}$ of symmetries of a regular $n$-gon, there are two-dimensional faithful irreps. If $n$ is odd, there is one isotropy subgroup isomorphic to $Z_{2}$, while if $n$ is even there are two, corresponding to the one or two non-conjugate reflection symmetries [11].

Another example of an analytical result is available for the symmetric group $S_{n}$, where the natural $n$-dimensional representation is reducible and composed of the trivial irrep and an irrep of dimension $n-1$. Here, the isotropy subgroups are of the form $S_{p} \times S_{n-p}$ for $p=1 \ldots[n / 2]$, corresponding to a partition of $n$ identical objects into two classes of size $p$ and $n-p$ [10]. There is an analogous result for the alternating group $A_{n}$ of even permutations. For $n \geqslant 4$, there is a natural irrep of dimension $n-1$, with [ $n / 2]$ isotropy subgroups that are the stabilisers of the same partition. The subgroups are generated by even permutations of one subset and odd permutations of both subsets, so for $p=1$ the axial isotropy subgroup is $A_{n-1}$, and for $p=2$ it is isomorphic to $S_{n-2}$ (since any permutation of $n-2$ objects can be written as an even permutation of $n$ objects, by interchanging the remaining two objects, or not, as required).

\subsection{Symmetric, alternating and Platonic groups}

In this section, results are presented in tabular form for some symmetric groups, alternating groups and the symmetry groups of the Platonic solids.

The results are given in the form of extended character tables for each group $G$. The first row of each table gives the number of elements in each conjugacy class, and the second row gives the orders of the elements in that class. Each subsequent row corresponds to a numbered irrep of $G$, marked with a symbol ' $F$ ' if the irrep is faithful, and gives the character for each class. This is followed by lists of isotropy subgroups with $D(H)=1$ and $D(H)=2$. The subgroups are labelled according to isotropy; in some cases, this does not completely define the subgroup, but to specify the group completely, by specifying its generators, would make the tables unwieldy. Larger groups that cannot easily be labelled are simply denoted $H_{n}$, where $n=|H|$. For isotropy subgroups with $N(H) \neq H$, the size of the quotient group $N(H) / H$ is given in brackets after the subgroup. Thus, if a group 
with $D(H)=1$ is followed by (2), the corresponding bifurcation must be a pitchfork. For subgroups with $D(H)=2$, the subgroup is followed by the symbol ' $\mathrm{M}$ ' if the subgroup is maximal (recall that all isotropy subgroups with $D(H)=1$ are maximal).

For the alternating group $A_{4}$ (Table 2), which is also the symmetry group of rotations of a tetrahedron, there is one faithful 3D irrep, with axial subgroups $Z_{2}$ and $Z_{3}$, corresponding to the two possible rotation axes of the tetrahedron.

The group $A_{5}$ is isomorphic to the rotation group of the icosahedron or dodecahedron (see Table 3). The problem of bifurcation with icosahedral symmetry has recently been investigated by Hoyle [12], who also computed the normal form equations for the bifurcation in each irrep, and derived stability conditions for each axial branch of solutions. For the $3 \mathrm{D}$ irreps, there are axial subgroups $Z_{2}, Z_{3}$ and $Z_{5}$, each corresponding to a rotation axis of the icosahedron, and in the natural 4D irrep there are axial subgroups $D_{3} \equiv S_{3}$ and $A_{4}$, in agreement with the general result for $A_{n}$ given above. Note that in the $5 \mathrm{D}$ irrep, there is a maximal isotropy subgroup $D_{2}$ with $D(H)=2$; it can be shown using the equations in $[12]$ and the methods of $[\mathbf{1 5}, \mathbf{1 6}, \mathbf{1 8}]$ that solutions with isotropy $D_{2}$ exist, in addition to the $D_{3}$ and $D_{5}$ solutions guaranteed by the equivariant branching lemma.

For the group $A_{6}$ (Table 4), there are several cases where the equivariant branching lemma indicates branching to solutions with isomorphic but non-conjugate isotropy subgroups. In the natural 5D irrep, there are solutions with isotropy $A_{5}$ and $S_{4}$, and an 18-element group generated by the permutations $(1,2,3),(4,5,6)$ and $(1,2)(4,5)$, as discussed above. In the 10D irrep, there are three maximal isotropy subgroups with $D(H)=2$. Results have also been obtained for $A_{7}$ and $A_{8}$ (of order 20160 ), where there are up to 21 axial subgroups.

Table 2: Results for the group $A_{4}$, the group of rotations of a tetrahedron (see the beginning of Section 4.1 for notation).

\begin{tabular}{l|rrrr|ll}
\hline & 1 & 3 & 4 & 4 & & \\
& 1 & 2 & 3 & 3 & $D(H)=1$ & $D(H)=2$ \\
\hline 1 & 1 & 1 & 1 & 1 & $A_{4}$ & \\
2 & 1 & 1 & $e^{2 \pi i / 3}$ & $e^{-2 \pi i / 3}$ & $D_{2}$ & \\
3 & 1 & 1 & $e^{-2 \pi i / 3}$ & $e^{2 \pi i / 3}$ & $D_{2}$ & \\
$4 \mathrm{~F}$ & 3 & -1 & 0 & 0 & $Z_{2}(2) Z_{3}$ & \\
\hline
\end{tabular}

Table 3: Results for the group $A_{5}$, the group of rotations of an icosahedron; the symbols $\phi_{ \pm}$ denote $(1 \pm \sqrt{5}) / 2$.

\begin{tabular}{l|rrrrr|ll}
\hline & 1 & 15 & 20 & 12 & 12 & & \\
& 1 & 2 & 3 & 5 & 5 & $D(H)=1$ & $D(H)=2$ \\
\hline 1 & 1 & 1 & 1 & 1 & 1 & $A_{5}$ & \\
$2 \mathrm{~F}$ & 3 & -1 & 0 & $\phi_{+}$ & $\phi_{-}$ & $Z_{2}(2) Z_{3}(2) Z_{5}(2)$ & \\
$3 \mathrm{~F}$ & 3 & -1 & 0 & $\phi_{-}$ & $\phi_{+}$ & $Z_{2}(2) Z_{3}(2) Z_{5}(2)$ & \\
$4 \mathrm{~F}$ & 4 & 0 & 1 & -1 & -1 & $D_{3} A_{4}$ & $Z_{2}(2) Z_{3}(2)$ \\
$5 \mathrm{~F}$ & 5 & 1 & -1 & 0 & 0 & $D_{3} D_{5}$ & $D_{2} \mathrm{M}(3)$ \\
\hline
\end{tabular}


Turning to the symmetric groups, Table 5 shows the case of $S_{4}$, which has been discussed in earlier work $[\mathbf{1 1}, \mathbf{1 9}]$. This group is isomorphic to the group of rotation symmetries of the cube (or octahedron), and also to the group of symmetries of the tetrahedron, including reflections. The 'natural' irrep for the rotations of the cube is that labelled $4 \mathrm{~F}$, and the axial subgroups correspond to rotations about the two-fold, three-fold and four-fold axes of the cube. Irrep $5 \mathrm{~F}$ is the 'natural' one for the tetrahedron, or for the permutation of four objects. The isotropy subgroups $D_{2}$ and $D_{3}$ are analogous to the rotation axes found for $A_{4}$, and the subgroup $Z_{2}$ with $D(H)=2$ is generated by the reflection symmetry of the tetrahedron.

Table 4: Results for the group $A_{6}$. The symbols $\phi_{ \pm}$denote $(1 \pm \sqrt{5}) / 2$.

\begin{tabular}{l|rrrrrrr}
\hline & 1 & 45 & 40 & 40 & 90 & 72 & 72 \\
& 1 & 2 & 3 & 3 & 4 & 5 & 5 \\
\hline 1 & 1 & 1 & 1 & 1 & 1 & 1 & 1 \\
$2 \mathrm{~F}$ & 5 & 1 & 2 & -1 & -1 & 0 & 0 \\
$3 \mathrm{~F}$ & 5 & 1 & -1 & 2 & -1 & 0 & 0 \\
$4 \mathrm{~F}$ & 8 & 0 & -1 & -1 & 0 & $\phi_{+}$ & $\phi_{-}$ \\
$5 \mathrm{~F}$ & 8 & 0 & -1 & -1 & 0 & $\phi_{-}$ & $\phi_{+}$ \\
$6 \mathrm{~F}$ & 9 & 1 & 0 & 0 & 1 & -1 & -1 \\
$7 \mathrm{~F}$ & 10 & -2 & 1 & 1 & 0 & 0 & 0 \\
\hline
\end{tabular}

\begin{tabular}{l|ll}
\hline & $D(H)=1$ & $D(H)=2$ \\
\hline 1 & $A_{6}$ & \\
$2 \mathrm{~F}$ & $H_{18}(2) S_{4} A_{5}$ & $D_{2}(6) D_{3} A_{4}(2)$ \\
$3 \mathrm{~F}$ & $H_{18}(2) S_{4} A_{5}$ & $D_{2}(6) D_{3} A_{4}(2)$ \\
$4 \mathrm{~F}$ & $D_{3} D_{3} D_{4} D_{5}$ & $Z_{3}(6) Z_{3}(6) D_{2}(6) D_{2}(6) Z_{4}(2) Z_{5}(2)$ \\
$5 \mathrm{~F}$ & $D_{3} D_{3} D_{4} D_{5}$ & $Z_{3}(6) Z_{3}(6) D_{2}(6) D_{2}(6) Z_{4}(2) Z_{5}(2)$ \\
$6 \mathrm{~F}$ & $D_{5} S_{4} S_{4} H_{36}$ & $D_{3} D_{3} D_{4}$ \\
$7 \mathrm{~F}$ & $D_{3} D_{3} A_{4}(2) A_{4}(2)$ & $Z_{4} \mathrm{M}(2) Z_{5} \mathrm{M}(2) Z_{3} \times Z_{3} \mathrm{M}(4)$ \\
\hline
\end{tabular}

Table 5: Results for the symmetric group $S_{4}$.

\begin{tabular}{l|rrrrr|ll}
\hline & 1 & 6 & 3 & 8 & 6 & & \\
& 1 & 2 & 2 & 3 & 4 & $D(H)=1$ & $D(H)=2$ \\
\hline 1 & 1 & 1 & 1 & 1 & 1 & $S_{4}$ & \\
2 & 1 & -1 & 1 & 1 & -1 & $A_{4}(2)$ & \\
3 & 2 & 0 & 2 & -1 & 0 & $D_{4}$ & $D_{2}(6)$ \\
$4 \mathrm{~F}$ & 3 & -1 & -1 & 0 & 1 & $Z_{2}(2) Z_{3}(2) Z_{4}(2)$ & \\
$5 \mathrm{~F}$ & 3 & 1 & -1 & 0 & -1 & $D_{2}(2) D_{3}$ & $Z_{2}(2)$ \\
\hline
\end{tabular}


For $S_{5}$ (see Table 6), the natural permutation irrep is 4F, giving axial subgroups $S_{4}$ and $S_{3} \times S_{2} \equiv D_{6}$. Results have also been found for $S_{6}, S_{7}$ and $S_{8}$.

The remaining symmetry groups for the Platonic solids are the groups $S_{4} \times Z_{2}$ for the symmetry of the cube including reflections (Table 7), and $A_{5} \times Z_{2}$ for the symmetry of the icosahedron with reflections (Table 8). The results are consistent with the earlier work $[19,12]$ in each case. For $S_{4} \times Z_{2}$, the two faithful 3D irreps lead to axial subgroups $D_{2}$, $D_{3}$ and $D_{4}$. For $A_{5} \times Z_{2}$, in the faithful 5D irrep there are axial subgroups $D_{2}, D_{3}$ and $D_{5}$; as in the case of $A_{5}$, there is a maximal two-dimensional isotropy subgroup $D_{2}$. In this case, however, it can be shown (using the equations given in [12]) that there is generically no solution in this subspace.

Note that for a group $G$ with a direct product structure $G=K \times Z_{2}$, the irreps can be obtained from those for the group $K$; each irrep of $K$ corresponds to two irreps ' + ' and ' - ' of $G$ according to the action of the $Z_{2}$ subgroup. For the ' + ' irreps, which are not faithful, the isotropy subgroups are simply the direct products of the isotropy subgroups for $K$ with $Z_{2}$, but for the faithful '-' irreps there is no simple relationship between the isotropy subgroups of $K$ and those of $G$.

Table 6: Results for the symmetric group $S_{5}$.

\begin{tabular}{l|rrrrrrr}
\hline & 1 & 10 & 15 & 20 & 20 & 30 & 24 \\
& 1 & 2 & 2 & 3 & 6 & 4 & 5 \\
\hline 1 & 1 & 1 & 1 & 1 & 1 & 1 & 1 \\
2 & 1 & -1 & 1 & 1 & -1 & -1 & 1 \\
$3 \mathrm{~F}$ & 4 & -2 & 0 & 1 & 1 & 0 & -1 \\
$4 \mathrm{~F}$ & 4 & 2 & 0 & 1 & -1 & 0 & -1 \\
$5 \mathrm{~F}$ & 5 & -1 & 1 & -1 & -1 & 1 & 0 \\
$6 \mathrm{~F}$ & 5 & 1 & 1 & -1 & 1 & -1 & 0 \\
$7 \mathrm{~F}$ & 6 & 0 & -2 & 0 & 0 & 0 & 1 \\
\hline
\end{tabular}

\begin{tabular}{l|ll}
\hline & $D(H)=1$ & $D(H)=2$ \\
\hline 1 & $S_{5}$ & \\
2 & $A_{5}(2)$ & \\
$3 \mathrm{~F}$ & $Z_{4}(2) D_{3}(2) Z_{6}(2) A_{4}(2)$ & $Z_{2}(4) Z_{3}(4)$ \\
$4 \mathrm{~F}$ & $D_{6} S_{4}$ & $D_{2}(2) D_{3}(2)$ \\
$5 \mathrm{~F}$ & $D_{3}(2) D_{4} H_{20}$ & $Z_{2}(6) D_{2}(6) Z_{4}(2)$ \\
$6 \mathrm{~F}$ & $D_{4} D_{5}(2) D_{6}$ & $D_{2}(6) D_{2}(2)$ \\
$7 \mathrm{~F}$ & $Z_{4}(2) D_{2}(2) D_{3}(2) Z_{6}(2)$ & $Z_{2}(4) Z_{3}(4) Z_{5} \mathrm{M}(4)$ \\
\hline
\end{tabular}


Table 7: Results for the group $S_{4} \times Z_{2}$ of symmetries of the cube including reflections.

\begin{tabular}{l|rrrrrrrrrr}
\hline & 1 & 1 & 6 & 6 & 8 & 8 & 3 & 3 & 6 & 6 \\
& 1 & 2 & 2 & 2 & 3 & 6 & 2 & 2 & 4 & 4 \\
\hline 1 & 1 & 1 & 1 & 1 & 1 & 1 & 1 & 1 & 1 & 1 \\
2 & 1 & 1 & -1 & -1 & 1 & 1 & 1 & 1 & -1 & -1 \\
3 & 1 & -1 & 1 & -1 & 1 & -1 & 1 & -1 & 1 & -1 \\
4 & 1 & -1 & -1 & 1 & 1 & -1 & 1 & -1 & -1 & 1 \\
5 & 2 & 2 & 0 & 0 & -1 & -1 & 2 & 2 & 0 & 0 \\
6 & 2 & -2 & 0 & 0 & -1 & 1 & 2 & -2 & 0 & 0 \\
$7 \mathrm{~F}$ & 3 & -3 & -1 & 1 & 0 & 0 & -1 & 1 & 1 & -1 \\
$8 \mathrm{~F}$ & 3 & -3 & 1 & -1 & 0 & 0 & -1 & 1 & -1 & 1 \\
9 & 3 & 3 & -1 & -1 & 0 & 0 & -1 & -1 & 1 & 1 \\
10 & 3 & 3 & 1 & 1 & 0 & 0 & -1 & -1 & -1 & -1 \\
\hline
\end{tabular}

\begin{tabular}{l|ll}
\hline & $D(H)=1$ & $D(H)=2$ \\
\hline 1 & $H_{48}$ & \\
2 & $H_{24}(2)$ & \\
3 & $S_{4}(2)$ & \\
4 & $S_{4}(2)$ & \\
5 & $D_{4} \times Z_{2}(6)$ \\
6 & $D_{4}(2) D_{4}(2)$ & $D_{2}(12)$ \\
$7 \mathrm{~F}$ & $D_{2}(2) D_{3}(2) D_{4}(2)$ & $Z_{2}(4) Z_{2}(8)$ \\
$8 \mathrm{~F}$ & $D_{2}(2) D_{3}(2) D_{4}(2)$ & $Z_{2}(4) Z_{2}(8)$ \\
9 & $D_{2}(2) Z_{6}(2) Z_{4} \times Z_{2}(2)$ & \\
10 & $Z_{2}^{3}(2) D_{6}$ & $D_{2}(2)$ \\
\hline
\end{tabular}


Table 8: Results for the group $A_{5} \times Z_{2}$ of symmetries of the icosahedron, including reflections.

\begin{tabular}{|c|c|c|c|c|c|c|c|c|c|c|}
\hline & 1 & 1 & 20 & 20 & 15 & 15 & 12 & 12 & 12 & 12 \\
\hline & 1 & 2 & 3 & 6 & 2 & 2 & 5 & 10 & 5 & 10 \\
\hline 1 & 1 & 1 & 1 & 1 & 1 & 1 & 1 & 1 & 1 & 1 \\
\hline 2 & 1 & -1 & 1 & -1 & 1 & -1 & 1 & -1 & 1 & -1 \\
\hline 3 & 3 & 3 & 0 & 0 & -1 & -1 & $\phi_{+}$ & $\phi_{+}$ & $\phi_{-}$ & $\phi_{-}$ \\
\hline $4 \mathrm{~F}$ & 3 & -3 & 0 & 0 & -1 & 1 & $\phi_{+}$ & $-\phi_{+}$ & $\phi_{-}$ & $-\phi_{-}$ \\
\hline 5 & 3 & 3 & 0 & 0 & -1 & -1 & $\phi_{-}$ & $\phi_{-}$ & $\phi_{+}$ & $\phi_{+}$ \\
\hline $6 \mathrm{~F}$ & 3 & -3 & 0 & 0 & -1 & 1 & $\phi_{-}$ & $-\phi_{-}$ & $\phi_{+}$ & $-\phi_{+}$ \\
\hline 7 & 4 & 4 & 1 & 1 & 0 & 0 & -1 & -1 & -1 & -1 \\
\hline $8 \mathrm{~F}$ & 4 & -4 & 1 & -1 & 0 & 0 & -1 & 1 & -1 & 1 \\
\hline 9 & 5 & 5 & -1 & -1 & 1 & 1 & 0 & 0 & 0 & ( \\
\hline $10 \mathrm{~F}$ & 5 & -5 & -1 & 1 & 1 & -1 & 0 & 0 & 0 & ( \\
\hline & & \multicolumn{5}{|c|}{$D(H)=1$} & \multicolumn{4}{|c|}{$D(H)=2$} \\
\hline & & \multicolumn{9}{|c|}{$A_{5} \times Z_{2}$} \\
\hline 2 & 2 & \multicolumn{9}{|c|}{$A_{5}(2)$} \\
\hline 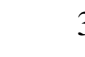 & 3 & \multicolumn{9}{|c|}{$D_{2}(2) Z_{6}(2) Z_{10}(2)$} \\
\hline & $4 \mathrm{~F}$ & \multicolumn{5}{|c|}{$D_{2}(2) D_{3}(2) D_{5}(2)$} & \multicolumn{4}{|c|}{$Z_{2}(4)$} \\
\hline 5 & 5 & \multicolumn{9}{|c|}{$D_{2}(2) Z_{6}(2) Z_{10}(2)$} \\
\hline & $6 \mathrm{~F}$ & \multicolumn{5}{|c|}{$D_{2}(2) D_{3}(2) D_{5}(2)$} & \multicolumn{4}{|c|}{$Z_{2}(4)$} \\
\hline 7 & 7 & \multicolumn{5}{|c|}{$D_{6} A_{4} \times Z_{2}$} & \multicolumn{4}{|c|}{$D_{2}(2) Z_{6}(2)$} \\
\hline & $3 F$ & \multicolumn{5}{|c|}{$D_{2}(2) D_{3}(2) D_{3}(2) A_{4}(2)$} & \multicolumn{4}{|c|}{$Z_{2}(4) Z_{2}(4) Z_{3}$} \\
\hline s & 9 & \multicolumn{5}{|c|}{$D_{6} D_{10}$} & \multicolumn{3}{|c|}{$Z_{2}^{3} \mathrm{M}$} & \\
\hline & $10 \mathrm{~F}$ & \multicolumn{5}{|c|}{$D_{2}(2) D_{3}(2) D_{5}(2)$} & \multicolumn{3}{|c|}{$Z_{2}(4) D_{2} \mathrm{M}(6)$} & \\
\hline
\end{tabular}




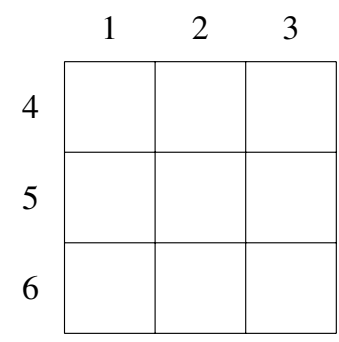

(a)

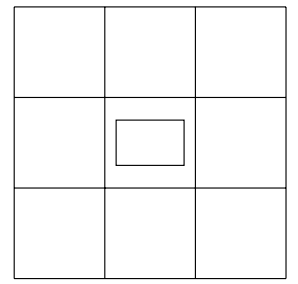

(d)

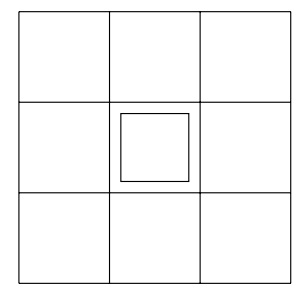

(b)

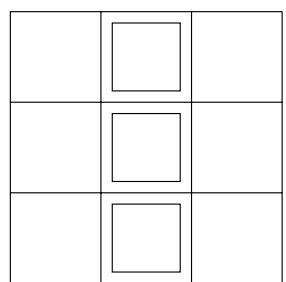

(c)

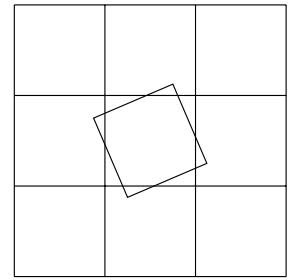

(e)

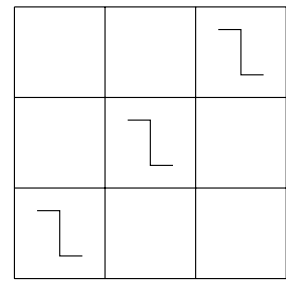

(f)

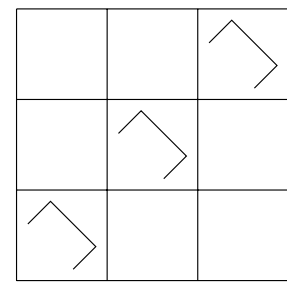

(g)

Figure 1: Symmetry-breaking from $D_{4} \ltimes Z_{3} \times Z_{3}$.

(a) The basic state and labelling used to write the group as a subgroup of $S_{6}$.

(b),(c) Schematic showing symmetry of axial solutions corresponding to irrep 6F with isotropy $D_{4}$ and $D_{6}$.

(d)-(g) Axial solutions corresponding to irrep 8F with isotropy $D_{2}, Z_{4}, D_{3}$ and $Z_{6}$.

\subsection{Instabilities of periodic patterns}

An application of considerable current interest is the classification of instabilities of regular, spatially periodic patterns that break the periodicity of the pattern but maintain periodicity on some larger lengthscale. The resulting patterns are known as superlattice patterns. The examples discussed here are motivated by recent observations of transitions seen in the Faraday experiment $[\mathbf{1}, \mathbf{1 4}]$. In this experiment, a layer of fluid or granular material is subjected to a vertical vibration. The uniform state can become unstable to a regular periodic pattern, which is often hexagonal. Also, by varying the parameters and introducing two-frequency forcing, a variety of secondary instabilities can be induced, leading to superlattice patterns. Since the underlying state in the Faraday experiment is periodic, the general theory of bifurcation from a stationary state outlined in Section 2 is not directly applicable. However, an analogous approach can be followed, in which the differential equations are replaced by maps describing the change in the state of the system after one cycle (see, for example, [24]).

Consider first a spatially periodic pattern of squares, with translation symmetries $x \rightarrow x+l$ and $y \rightarrow y+l$, and suppose that the symmetry of the pattern is broken in such a way that translation symmetries $x \rightarrow x+n l$ and $y \rightarrow y+n l, n \in \mathbb{N}$, are retained. With this constraint, the symmetry of the initial state is that of a periodic array of $n \times n$ squares, as shown in Figure 1(a) for $n=3$. The symmetry group is generated by the symmetries $D_{4}$ of the square, together with the symmetries $Z_{3} \times Z_{3}$ of the translations. Since the translations form a normal subgroup, the full symmetry group can be written as the semidirect product $G=D_{4} \ltimes Z_{3} \times Z_{3}$. This group can conveniently be written 
as a subgroup of the permutation group $S_{6}$, by labelling the three rows and three columns as shown in Figure 1(a), and by writing down the permutation action of the group generators on the rows and columns. The reflection about a vertical axis interchanges columns 1 and 3 , but fixes column 2 and the three rows, and so is represented by the permutation $(1,3)$. The reflection about the diagonal interchanges the rows and columns, corresponding to the permutation $(1,4)(2,5)(3,6)$. The translation $x \rightarrow x+l$ permutes the columns according to $(1,2,3)$ (the translation $y \rightarrow y+l$ is not required to generate the group). The results of the symmetry-breaking computation are shown in Table 9. Since the subgroup $Z_{3} \times Z_{3}$ is normal, the character table of the quotient group $D_{4}$ appears in the first five rows of the character table of $G$. These first five irreps correspond to instabilities of the square pattern that do not break any translation symmetries. There are four faithful four-dimensional irreps. In two of these, there are two axial subgroups, $D_{4}$ and $D_{6}$. Further examination of the generators of these subgroups shows that in irrep $6 \mathrm{~F}$, the first pattern retains the square symmetry $(1,3)$, $(1,4)(2,5)(3,6)$ but no translation symmetry (shown schematically in Figure 1(b)), while the $D_{6}$ solution possesses a translation symmetry $(4,5,6)$ and two orthogonal reflection symmetries $(1,3)$ and $(4,6)$ (see Figure $1(\mathrm{c})$ ). In irrep $7 \mathrm{~F}$, the $D_{4}$ solution is the same, but the $D_{6}$ solution involves diagonal translations and reflections. Thus the $D_{6}$ subgroups in Table 9 are not conjugate, but the $D_{4}$ subgroups are conjugate. In these irreps there are no normaliser symmetries, so we expect these patterns to arise at a transcritical bifurcation. In the other four-dimensional irreps there are four competing patterns, with isotropies $D_{2}$, $Z_{4}, D_{3}$ and $Z_{6}$. The solutions with $D_{2}$ and $Z_{4}$ symmetry have rectangular and $\pi / 2$ rotation symmetry, respectively, and no translation symmetry (see Figure 1(d), (e)). The patterns with $D_{3}$ and $Z_{6}$ possess a translation symmetry combined with a $\pi$ rotation symmetry and a reflection symmetry respectively (Figure $1(\mathrm{f}),(\mathrm{g})$ ). The $Z_{4}$ subgroups in irreps $8 \mathrm{~F}$ and $9 \mathrm{~F}$ are conjugate, but the $D_{2}, Z_{6}$ and $D_{3}$ subgroups are not; they are related through a $\pi / 4$ rotation. In these cases, the bifurcations are all pitchforks. In fact, the isotropy subgroups for $D_{4} \ltimes Z_{n} \times Z_{n}$ can be obtained analytically; the details of these calculations will be given elsewhere.

Table 9: Results for the group $D_{4} \ltimes Z_{3} \times Z_{3}$ of symmetries of a $3 \times 3$ square lattice.

\begin{tabular}{l|rrrrrrrrr|l}
\hline & 1 & 2 & 3 & 2 & 6 & 3 & 2 & 4 & 6 & $D(H)=1$ \\
\hline 1 & 1 & 1 & 1 & 1 & 1 & 1 & 1 & 1 & 1 & $D_{4} \ltimes Z_{3} \times Z_{3}$ \\
2 & 1 & -1 & 1 & 1 & -1 & 1 & 1 & -1 & 1 & $D_{2} \ltimes Z_{3} \times Z_{3}(2)$ \\
3 & 1 & 1 & 1 & 1 & 1 & 1 & -1 & -1 & -1 & $D_{2} \ltimes Z_{3} \times Z_{3}(2)$ \\
4 & 1 & -1 & 1 & 1 & -1 & 1 & -1 & 1 & -1 & $Z_{4} \ltimes Z_{3} \times Z_{3}(2)$ \\
5 & 2 & 0 & 2 & -2 & 0 & 2 & 0 & 0 & 0 & $Z_{2} \ltimes Z_{3} \times Z_{3}(2) Z_{2} \ltimes Z_{3} \times Z_{3}(2)$ \\
$6 \mathrm{~F}$ & 4 & 2 & 1 & 0 & -1 & -2 & 0 & 0 & 0 & $D_{4} D_{6}$ \\
$7 \mathrm{~F}$ & 4 & 0 & -2 & 0 & 0 & 1 & 2 & 0 & -1 & $D_{4} D_{6}$ \\
$8 \mathrm{~F}$ & 4 & -2 & 1 & 0 & 1 & -2 & 0 & 0 & 0 & $D_{2}(2) Z_{4}(2) D_{3}(2) Z_{6}(2)$ \\
$9 \mathrm{~F}$ & 4 & 0 & -2 & 0 & 0 & 1 & -2 & 0 & 1 & $D_{2}(2) Z_{4}(2) D_{3}(2) Z_{6}(2)$ \\
\hline
\end{tabular}




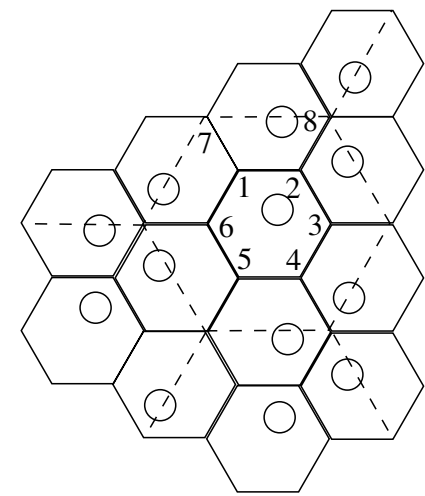

(a)

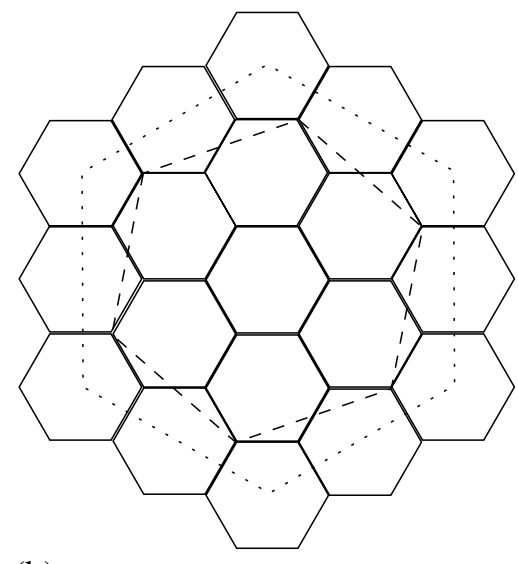

(b)

Figure 2: Symmetry-breaking on hexagonal lattices.

(a) Circles indicate qualitatively the symmetry of the 'SSS' state of [1]. The pattern is periodic on a larger hexagonal lattice, indicated by dashed lines. The numbered vertices are used to embed the group in $S_{8}$.

(b) For the superlattice patterns observed in [14], the pattern is periodic on a larger hexagonal lattice containing seven hexagons (SL-I, dashed line) or twelve hexagons (SL-II, dotted line) [24].

In the experiments of Arbell and Fineberg [1], square patterns are found in some parameter regimes, and these can become unstable to a pattern that appears to have square symmetry with a threefold increase in the periodicity (states referred to as ' $2 \mathrm{MS}$ ', see [1, Figure 2(b)]). The symmetry of these states is approximately as shown in Figure 1(b), so that the instability corresponds to the 4D irrep $6 \mathrm{~F}$ or $7 \mathrm{~F}$. There are axial solutions with $D_{4}$ and $D_{6}$ symmetry, but in the experiment it appears that only the $D_{4}$ solution is stable.

Arbell and Fineberg also observed hexagonal states, and these can become unstable to a state in which the hexagons cluster in groups of four ('SSS' states, see [1, Figure 2(a)]). This transition can be analysed by considering a hexagonal array set within a larger hexagonal lattice, so that each large hexagon contains four small hexagons (Figure 2(a)). In this case, the relevant symmetry group is $D_{6} \ltimes Z_{2} \times Z_{2}$, of order 48 , generated by the hexagonal symmetry and the two translations of the lattice. To write the group as a permutation group, the eight distinct vertices of the hexagons are labelled as shown in Figure 2. The generators are then the reflections $(1,2)(3,6)(4,5)(7,8)$ and $(1,3)(4,6)$ and the translation $(1,8)(2,6)(3,5)(4,7)$. Investigating the structure of the group reveals that it is isomorphic to the group of symmetries of the cube, $S_{4} \times Z_{2}$, the $Z_{2}$ subgroup being generated by a rotation through $\pi$ about the centre of a hexagon. Hence the possible symmetry-breaking instabilities are exactly analogous to those of the cube, and the axial subgroups can be read off from Table 7. The pattern observed in [1] and sketched in Figure 2(a) has $D_{2}$ symmetry generated by the reflections $(1,3)(4,6)$ and $(1,4)(2,8)(3,6)(5,7)$. The product of these reflections is a $\pi$ rotation about the edge of a hexagon. This subgroup is axial in the threedimensional faithful irreps $7 \mathrm{~F}$ and $8 \mathrm{~F}$ in Table 7. (Note that the pattern does not have the $D_{2}$ symmetry of irreps 9 and 10 , since in these irreps the $\pi$ rotation about the centre of a hexagon acts as the identity, so all the patterns have that symmetry.) 
In the Faraday experiments of Kudrolli, Pier and Gollub [14], two types of superlattice patterns were observed. The first type (SL-I) is periodic on a skewed hexagonal lattice containing seven hexagons, and the second (SL-II) is periodic on a lattice containing twelve hexagons (Figure 2(b)). The SL-II patterns were studied using the group-theoretic approach by Tse et al. [24]. In this case, the symmetry group can be embedded in $S_{12}$ simply by labelling the twelve hexagons and writing down the action of the two reflection symmetries and a translation symmetry. The translations generate the group $Z_{6} \times Z_{2}$, so the full spatial symmetry group of the system is $D_{6} \ltimes Z_{6} \times Z_{2}$, of order 144 (see [24]). The character table is shown in Table 10 (note that the character table given in [24] contains minor typographical

Table 10: Character table for the group $G=D_{6} \ltimes Z_{6} \times Z_{2}$ of symmetries of a periodic lattice of 12 hexagons, with axial subgroups for $G$ in irreps $14 \mathrm{~F}$ and $15 \mathrm{~F}$ and axial subgroups for $G \times Z_{2}$ in irrep $15 \mathrm{~F}-$.

\begin{tabular}{l|rrrrrrrrrrrrrrr}
\hline & 1 & 6 & 18 & 24 & 8 & 3 & 9 & 16 & 12 & 12 & 6 & 18 & 2 & 6 & 3 \\
& 1 & 2 & 2 & 6 & 3 & 2 & 2 & 3 & 12 & 6 & 6 & 4 & 3 & 4 & 2 \\
\hline 1 & 1 & 1 & 1 & 1 & 1 & 1 & 1 & 1 & 1 & 1 & 1 & 1 & 1 & 1 & 1 \\
3 & 1 & 1 & -1 & -1 & 1 & -1 & -1 & 1 & 1 & 1 & 1 & -1 & 1 & 1 & 1 \\
4 & 1 & -1 & -1 & 1 & 1 & 1 & 1 & 1 & -1 & -1 & 1 & -1 & 1 & -1 & 1 \\
5 & 2 & -1 & 1 & -1 & 1 & -1 & -1 & 1 & -1 & -1 & 1 & 1 & 1 & -1 & 1 \\
6 & 2 & 0 & 0 & -1 & -1 & 2 & 2 & -1 & 0 & 0 & 2 & 0 & 2 & 0 & 2 \\
7 & 2 & 2 & 0 & 0 & 2 & 0 & 0 & -1 & -1 & -1 & -1 & 0 & -1 & 2 & 2 \\
8 & 2 & -2 & 0 & 0 & 2 & 0 & 0 & -1 & 1 & 1 & -1 & 0 & -1 & -2 & 2 \\
9 & 3 & -1 & -1 & 0 & 0 & 3 & -1 & 0 & 1 & -1 & -1 & 1 & 3 & 1 & -1 \\
10 & 3 & -1 & 1 & 0 & 0 & -3 & 1 & 0 & 1 & -1 & -1 & -1 & 3 & 1 & -1 \\
11 & 3 & 1 & -1 & 0 & 0 & -3 & 1 & 0 & -1 & 1 & -1 & 1 & 3 & -1 & -1 \\
12 & 3 & 1 & 1 & 0 & 0 & 3 & -1 & 0 & -1 & 1 & -1 & -1 & 3 & -1 & -1 \\
13 & 4 & 0 & 0 & 0 & -2 & 0 & 0 & 1 & 0 & 0 & -2 & 0 & -2 & 0 & 4 \\
$14 \mathrm{~F}$ & 6 & -2 & 0 & 0 & 0 & 0 & 0 & 0 & -1 & 1 & 1 & 0 & -3 & 2 & -2 \\
$15 \mathrm{~F}$ & 6 & 2 & 0 & 0 & 0 & 0 & 0 & 0 & 1 & -1 & 1 & 0 & -3 & -2 & -2 \\
\hline
\end{tabular}

\begin{tabular}{l|l}
\hline $14 \mathrm{~F}$ & $D_{2}(2) D_{2}(2) Z_{6}(2) D_{3}(2) D_{3} D_{4}(2) Z_{4} \times Z_{2}(2)$ \\
$15 \mathrm{~F}$ & $D_{3} D_{4}(2) Z_{2}^{3}(2) D_{6}$ \\
$15 \mathrm{~F}-$ & $D_{3}(2) D_{3}(2) Z_{2}^{3}$ (2) $Z_{2}^{3}$ (2) $D_{6}$ (2) $D_{6}(2) D_{4} \times Z_{2}$ (2) $D_{4} \times Z_{2}(2)$ \\
\hline
\end{tabular}


errors that do not affect the results). In fact, the group is isomorphic to $S_{4} \times S_{3}$, although this is by no means obvious and does not generalise to other superlattices. Note that there are two faithful six-dimensional irreps. It is shown in [24] that the relevant irrep for the SL-II pattern of [14] is $15 \mathrm{~F}$. The axial subgroups for the faithful irreps $14 \mathrm{~F}$ and $15 \mathrm{~F}$ are listed in Table 10; there are four axial subgroups for irrep 15F.

An additional complication in this example is that the observed instability is subharmonic; that is, it arises at a period-doubling bifurcation, with Floquet multiplier -1 . It is explained in [24] that this can be incorporated by including an additional $Z_{2}$ symmetry to represent the time translation, so that the group is now isomorphic to $\left(D_{6} \ltimes Z_{6} \times Z_{2}\right) \times Z_{2}$, of order 288. As discussed at the end of Section 4.1, each irrep of the original group then splits into two irreps, ' + ' and '-', according to the action of the additional $Z_{2}$ symmetry. The irrep of interest is then the $15 \mathrm{~F}$ - irrep, and the axial subgroups for this irrep are listed in the last row of Table 10. Tse et al. [24] state that there are six axial isotropy subgroups for this irrep, but do not explain how they did the calculation. Since there are 272 subgroups, it is likely that the axial subgroups were found by inspection, rather than by following the systematic algorithm outlined in Table 1 . In fact, the computational algorithm reveals that there are eight axial subgroups. The pair of subgroups isomorphic to $D_{3}$ are omitted in [24]; the solution corresponding to the SL-II pattern of [14] is identified in [24] as one of the solutions with isotropy $D_{4} \times Z_{2}$. Most of the axial subgroups are 'twisted'; that is, they include spatiotemporal symmetries involving a spatial symmetry and a time shift. However, one of the $D_{3}$ solutions and one of the $D_{6}$ solutions correspond to states with purely spatial symmetries. These states have triangular symmetry about a vertex of one of the original hexagons, and hexagonal symmetry about the centre of one of the original hexagons, respectively.

\section{Oscillatory bifurcations}

The preceding sections have been concerned entirely with the case of a stationary bifurcation, where a real eigenvalue passes through zero, leading to branches of stationary solutions. We now consider the case of an oscillatory, or Hopf, bifurcation, where a complex conjugate pair of eigenvalues crosses into the right half-plane and the bifurcating branches are periodic orbits.

For the case of a Hopf bifurcation, it is necessary to consider symmetry-breaking from the group $G \times S^{1}$, where $S^{1}$ denotes the continuous time symmetry that is broken in the Hopf bifurcation (see $[\mathbf{9}, \mathbf{1 0}, \mathbf{1 1}]$ for details of the general theory). The resulting solution branches have spatiotemporal symmetries, involving combinations of elements of $G$ with time translations. More precisely, the spatiotemporal symmetry $H$ contains a normal subgroup $K$ that is an isotropy subgroup of $G$, and $H / K$ is a cyclic group representing the time translations. This cyclic group $H / K$ must be maximally abelian in $N(K) / K$. These conditions are necessary for the Hopf bifurcation form of the equivariant branching lemma to be valid [9]. For the dihedral group $D_{n}$, in the two-dimensional irreps, it is well known that there are three solution branches [11]. The subgroups $H$ (with spatial subgroup $K$ in brackets) are $Z_{n}(1), Z_{2}(1), Z_{2}\left(Z_{2}\right)$ for $n$ odd, and $Z_{n}(1), D_{2}\left(Z_{2}\right), D_{2}\left(Z_{2}\right)$ for $n$ even.

Since the time translation symmetry is a cyclic group, a convenient algorithm for reducing the case of the Hopf bifurcation to the case of the stationary bifurcation is to consider the finite group $G \times Z_{m}$, for some choice of $m$. It is straightforward to see that the time shift symmetry $H / K$ must have an order that is equal the order of one of the elements of $G$, so $m$ can be chosen as the least common multiple of the orders of the elements of $G$. 
For example, $m=4$ for $D_{4}, m=12$ for $S_{4}$, and $m=30$ for $A_{5}$. In the cases when $m$ is large, an alternative approach is to consider the different possible values of $m$ separately; for example, for $A_{5}$, the calculation is repeated for $A_{5} \times Z_{2}, A_{5} \times Z_{3}$ and $A_{5} \times Z_{5}$.

The results obtained using this algorithm are listed in Table 11 . For each group, only the faithful irreps are considered, and these are listed using the same labelling as in the tables in Section 4 for the stationary bifurcation. For each solution, the full spatiotemporal symmetry $H$ is given, as well as the normal subgroup $K$ representing the spatial part. In some cases $H=K$, indicating that the symmetries of the periodic orbit are purely spatial.

In a few cases, the results in Table 11 had been obtained previously. Swift and Barany [23] considered Hopf bifurcation with the symmetry of rotation of a tetrahedron $\left(A_{4}\right)$. They found the four branches of periodic solutions shown in Table 11, and showed that the normal form equations can exhibit chaotic behaviour. For the group $S_{4}$, which is the group of rotations of a cube, Ashwin and Podvigina [2] studied the Hopf bifurcation in irrep 4F; their results are in agreement with Table 11. In irrep 5F, the axial solutions have isotropy that is isomorphic to those for irrep 4F. This is because the characters for these two irreps differ only in signs (Table 5), and these signs can be changed by a half shift in time. Thus, although the spatiotemporal symmetry groups are isomorphic, the spatial subgroups are not.

Table 11: Axial solution branches for Hopf bifurcation. Labels for the irreps are as used on earlier tables for the stationary bifurcation. The spatiotemporal symmetry for each axial solution is given, with the spatial subgroup $K$ in brackets.

\begin{tabular}{l|l|l}
\hline Group & Irrep & Axial branches \\
\hline$A_{4}$ & $4 \mathrm{~F}$ & $Z_{3}(1) Z_{3}(1) Z_{3}\left(Z_{3}\right) D_{2}\left(Z_{2}\right)$ \\
$A_{5}$ & $2 \mathrm{~F}$ & $Z_{3}(1) Z_{5}(1) D_{2}\left(Z_{2}\right) D_{3}\left(Z_{3}\right) D_{5}\left(Z_{5}\right)$ \\
$A_{5}$ & $3 \mathrm{~F}$ & $Z_{3}(1) Z_{5}(1) D_{2}\left(Z_{2}\right) D_{3}\left(Z_{3}\right) D_{5}\left(Z_{5}\right)$ \\
$A_{5}$ & $4 \mathrm{~F}$ & $Z_{3}(1) Z_{5}(1) Z_{5}(1) D_{2}\left(Z_{2}\right) D_{3}\left(Z_{3}\right) D_{3}\left(D_{3}\right) A_{4}\left(A_{4}\right)$ \\
$A_{5}$ & $5 \mathrm{~F}$ & $Z_{5}(1) Z_{5}(1) D_{2}\left(Z_{2}\right) D_{3}\left(D_{3}\right) D_{5}\left(D_{5}\right) A_{4}\left(D_{2}\right) A_{4}\left(D_{2}\right)$ \\
$S_{4}$ & $4 \mathrm{~F}$ & $Z_{3}(1) Z_{4}(1) D_{2}\left(Z_{2}\right) D_{3}\left(Z_{3}\right) D_{4}\left(Z_{4}\right)$ \\
$S_{4}$ & $5 \mathrm{~F}$ & $Z_{3}(1) Z_{4}(1) D_{2}\left(Z_{2}\right) D_{3}\left(D_{3}\right) D_{4}\left(D_{2}\right)$ \\
$S_{4} \times Z_{2}$ & $7 \mathrm{~F}$ & $Z_{2}^{3}\left(D_{2}\right) Z_{6}(1) Z_{4} \times Z_{2}\left(Z_{2}\right) D_{6}\left(D_{3}\right) D_{4} \times Z_{2}\left(D_{4}\right)$ \\
$S_{4} \times Z_{2}$ & $8 \mathrm{~F}$ & $Z_{2}^{3}\left(D_{2}\right) Z_{6}(1) Z_{4} \times Z_{2}\left(Z_{2}\right) D_{6}\left(D_{3}\right) D_{4} \times Z_{2}\left(D_{4}\right)$ \\
$A_{5} \times Z_{2}$ & $4 \mathrm{~F}$ & $Z_{2}^{3}\left(D_{2}\right) D_{6}\left(D_{3}\right) D_{10}\left(D_{5}\right) Z_{6}(1) Z_{10}(1)$ \\
$A_{5} \times Z_{2}$ & $6 \mathrm{~F}$ & $Z_{2}^{3}\left(D_{2}\right) D_{6}\left(D_{3}\right) D_{10}\left(D_{5}\right) Z_{6}(1) Z_{10}(1)$ \\
$A_{5} \times Z_{2}$ & $8 \mathrm{~F}$ & $Z_{2}^{3}\left(D_{2}\right) D_{6}\left(D_{3}\right) D_{6}\left(D_{3}\right) A_{4} \times Z_{2}\left(A_{4}\right) Z_{6}(1) Z_{10}(1) Z_{10}(1)$ \\
$A_{5} \times Z_{2}$ & $10 \mathrm{~F}$ & $Z_{2}^{3}\left(D_{2}\right) D_{6}\left(D_{3}\right) D_{10}\left(D_{5}\right) A_{4} \times Z_{2}\left(D_{2}\right) A_{4} \times Z_{2}\left(D_{2}\right) Z_{10}(1) Z_{10}(1)$ \\
\hline
\end{tabular}


For the Hopf bifurcation with the symmetry of the cube including reflections, there are again five branches, and the two faithful irreps $7 \mathrm{~F}$ and $8 \mathrm{~F}$ of Table 7 give the same results. For Hopf bifurcation with icosahedral symmetry, there are seven distinct branches of periodic solutions in the four- and five-dimensional irreps. The calculation was also carried out for the group $D_{4} \ltimes Z_{3} \times Z_{3}$ (Figure 1), corresponding to an oscillatory instability of a regular square pattern. In this case, there are nine solution branches for each of the faithful four-dimensional irreps of Table 9.

Acknowledgements. I am grateful to Peter Ashwin, John Cremona, the GAP group and the referees for helpful advice and discussions.

\section{References}

1. H. Arbell and J. Fineberg, 'Spatial and temporal dynamics of two interacting modes in parametrically driven surface waves', Phys. Rev. Lett. 81 (1998) 4384-4387. 102, 112,114

2. P. Ashwin and O. Podvigina, 'Hopf bifurcation with cubic symmetry and instability of ABC flow', Proc. Roy. Soc. A 459 (2003) 1801-1827. 106, 117

3. E. Bodenschatz, W. Pesch and G. Ahlers, 'Recent developments in RayleighBénard convection', Ann. Rev. Fluid. Mech. 32 (2000) 709-778. 102

4. P. Chossat and R. LAUterbach, Methods in equivariant bifurcations and dynamical systems, Adv. Ser. Nonlin. Dynam. 15 (World Scientific, River Edge, NJ, 2000). 101, 102,103

5. G. Cicogna, 'Symmetry breakdown from bifurcations', Lettere al Nuovo Cimento 31 (1981) 600-602. 103

6. S. M. Cox and P. C. Matthews, 'Instability and localisation of patterns due to a conserved quantity’, Physica D 175 (2003) 196-219. 102

7. J. H. P. Dawes, P. C. Matthews and A. M. Rucklidge, 'Reducible actions of $D_{4} \ltimes T^{2}$ : superlattice patterns and hidden symmetries', Nonlinearity 16 (2003) 615-645. 102, 103

8. The GAP Group, GAP - Groups, algorithms, and programming, Version 4.3 (2002); http://www.gap-system.org. 102, 104

9. M. Golubitsky and I. Stewart, 'An algebraic criterion for symmetric Hopf bifurcation', Proc. Roy. Soc. London Ser. A 440 (1993) 727-732. 116

10. M. Golubitsky and I. STEWART, The symmetry perspective (Birkhäuser, Basel, 2002). $101,103,106,116$

11. M. Golubitsky, I. Stewart and D. G. Schaeffer, Singularities and groups in bifurcation theory, vol. II, Appl. Math. Ser. 69 (Springer, New York, 1988). 101, 102, 103, 105, 106, 108, 116

12. R. B. Hoyle, 'Shapes and cycles arising at the steady bifurcation with icosahedral symmetry', Physica D 191 (2004) 261-281. 106, 107, 109

13. E. Ihrig and M. Golubitsky, 'Pattern selection with $O(3)$ symmetry', Physica $D 13$ (1984) 1-33. 103, 105

14. A. Kudrolli, B. Pier and J. P. Gollub, 'Superlattice patterns in surface waves', Physica D 123 (1998) 99-111. 102, 112, 114, 115, 116 
15. A. LARI-LAVASSANI, W. F. LANGFORD and K. HuSEYIN, 'Symmetry-breaking bifurcations on multidimensional fixed point subspaces', Dynam. Stability Systems 9 (1994) 345-373. 103, 107

16. R. LAUterbaCH, 'Spontaneous symmetry breaking in higher-dimensional fixed point spaces', Z. Angew. Math. Phys. 43 (1992) 430-448. 103, 107

17. M. J. Linehan and G. E. Stedman, 'Little groups of irreps of $O(3), \mathrm{SO}(3)$, and the infinite axial subgroups', J. Phys. A 34 (2001) 6663-6688. 105

18. P. C. Matthews, 'Bifurcation in two-dimensional fixed point subspaces', preprint (2003); arXiv:math.DS/0305392. 103, 107

19. I. Melbourne, 'A singularity theory analysis of bifurcation problems with octahedral symmetry', Dynamics and Stability of Systems 1 (1986) 293-321. 106, 108, 109

20. M. Michel, 'Symmetry defects and broken symmetry', Rev. Modern Phys. 52 (1980) 617-651. 105

21. LoRd RAYleigh, 'On convection currents in a horizontal layer of fluid, when the higher temperature is on the underside', Philos. Mag. 32 (1916) 529-546. 102

22. D. H. SATTINGER, Group theoretic methods in bifurcation theory (Springer, New York, 1979). 101

23. J. W. SWIFT and E. BARANY, 'Chaos in the Hopf bifurcation with tetrahedral symmetry: convection in a rotating fluid with low Prandtl number', Proc., Nonlinear Hydrodynamic Stability and Transition, Sophia-Antipolis, 1990, European J. Mech. B Fluids 10 (1991) 99-104. 117

24. D. P. Tse, A. M. Rucklidge, R. B. Hoyle and M. Silber, 'Spatial period-multiplying instabilities of hexagonal Faraday waves', Physica D 146 (2000) 367-387. 102, 112, $114,115,116$

25. A. VANDERBAuWhede, 'Symmetry and bifurcation near families of solution', J. Differential Equations 36 (1980) 173-178. 103

P. C. Matthews paul.matthews@nottingham.ac.uk

http://www.maths.nottingham.ac.uk/personal/pcm

School of Mathematical Sciences

University of Nottingham

Nottingham NG7 2RD

United Kingdom 